\title{
BMJ Open Long-term use and cost-effectiveness of secondary prevention drugs for heart disease in Western Australian seniors (WAMACH): a study protocol
}

\author{
Anthony S Gunnell, ${ }^{1}$ Matthew W Knuiman, ${ }^{1}$ Elizabeth Geelhoed, ${ }^{1}$ \\ Michael S T Hobbs, ${ }^{1}$ Judith M Katzenellenbogen, ${ }^{2}$ Joseph Hung, ${ }^{3}$ \\ Jamie M Rankin, ${ }^{4}$ Lee Nedkoff, ${ }^{1}$ Thomas G Briffa, ${ }^{1}$ Michael Ortiz, ${ }^{5}$ Malcolm Gillies, ${ }^{6}$ \\ Anne Cordingley, ${ }^{7}$ Mitch Messer, ${ }^{8}$ Christian Gardner, ${ }^{1}$ Derrick Lopez, ${ }^{2}$ \\ Emily Atkins, ${ }^{1}$ Qun Mai, ${ }^{9}$ Frank M Sanfilippo ${ }^{1}$
}

To cite: Gunnell AS, Knuiman MW, Geelhoed E, et al. Long-term use and cost-effectiveness of secondary prevention drugs for heart disease in Western Australian seniors (WAMACH): a study protocol. BMJ Open 2014;4:e006258. doi:10.1136/bmjopen-2014006258

- Prepublication history and additional material is available. To view please visit the journal (http://dx.doi.org/ 10.1136/bmjopen-2014006258).

Received 30 July 2014 Accepted 15 August 2014

CrossMark

For numbered affiliations see end of article.

Correspondence to Dr Anthony S Gunnell; anthony.gunnell@uwa.edu.au

\section{ABSTRACT}

Introduction: Secondary prevention drugs for cardiac disease have been demonstrated by clinical trials to be effective in reducing future cardiovascular and mortality events (WAMACH is the Western Australian Medication Adherence and Costs in Heart disease study). Hence, most countries have adopted health policies and guidelines for the use of these drugs, and included them in government subsidised drug lists to encourage their use. However, suboptimal prescribing and nonadherence to these drugs remains a universal problem. Our study will investigate trends in dispensing patterns of drugs for secondary prevention of cardiovascular events and will also identify factors influencing these patterns. It will also assess the clinical and economic consequences of non-adherence and the costeffectiveness of using these drugs.

Methods and analysis: This population-based cohort study will use longitudinal data on almost 40000 people aged 65 years or older who were hospitalised in Western Australia between 2003 and 2008 for coronary heart disease, heart failure or atrial fibrillation. Linking of several State and Federal government administrative data sets will provide person-based information on drugs dispensed precardiac and postcardiac event, reasons for hospital admission, emergency department visits, mortality and medical visits. Dispensed drug trends will be described, drug adherence measured and their association with future all-cause/cardiovascular events will be estimated. The cost-effectiveness of these longterm therapies for cardiac disease and the impact of adherence will be evaluated.

Ethics and dissemination: Human Research Ethics Committee (HREC) approvals have been obtained from the Department of Health (Western Australian \#2011/ 62 and Federal) and the University of Western Australia (RA/4/1/1130), in addition to HREC approvals from all participating hospitals. Findings will be published in peer-reviewed medical journals and presented at local, national and international conferences. Results will also be disseminated to consumer groups.
Strengths and limitations of this study

- Population-wide, longitudinal coverage of cardiac-related hospitalisations and drugs dispensed for secondary prevention will enable generalisability, high statistical power and long and near-complete follow-up to measure effectiveness of drug use and adherence on survival and other outcomes.

- Access to linked precardiac and postcardiac event data allows us to identify incident cardiac events and existing comorbidities.

- Use of linked data will allow estimation of costs related to pharmaceutical and Medicare services, hospitalisation and health service utilisation following hospital discharge.

- It is not known whether patients used their dispensed medicines. Therefore, the use of drug dispensing data for assessing adherence assumes consumption of drugs following dispensing.

- Data are not available on the reason for being dispensed a drug, nor the dose and frequency, so the quantity taken per day will be unknown. However, the drug strength is known.

\section{INTRODUCTION}

Despite the existence of proven secondary prevention therapies in cardiac disease, there remains a high rate of new or recurrent cardiovascular events in those with prevalent disease. $^{1-3}$ This highlights the welldocumented evidence-treatment gap in this population, despite compelling evidence of benefits from drug therapy in preventing recurrent coronary heart disease (CHD), heart failure (HF) and atrial fibrillation (AF)-related stroke. These are overlapping cardiac conditions with acute and chronic manifestations, and are age-related and 
common diseases that impose a large cost burden on society, governments and individuals. The pharmacotherapy evidence for preventing new or recurrent cardiac events relates to four groups of drugs that improve outcomes: antiplatelets (eg, aspirin, P2Y12 inhibitors, dual antiplatelet therapy), $\beta$-blockers, lipid-lowering drugs (mostly statins) and ACE inhibitors (ACEI) or angiotensin II receptor blockers (ARB). ${ }^{1-3}$ For HF, the main drugs that improve outcomes are B-blockers, ACEI/ARB and aldosterone antagonists, ${ }^{3}$ while warfarin is used in $\mathrm{AF}^{4}$ Supporting the use of these medications for secondary prevention are the Heart Foundation and the Cardiac Society of Australia and New Zealand, which published guidelines on reducing risk in heart disease. ${ }^{3-6}$

The use of evidence-based medication on discharge from public and private hospitals in Western Australia (WA) in patients with acute coronary syndrome (ACS) has increased significantly over the past decade. A recent study using data from 2012 observed that $65 \%$ of Australian and New Zealand patients discharged from hospital following ACS were prescribed 4-5 of the recommended drugs for ACS. $^{7}$ Historical data from WA suggested that $50 \%$ of patients with ACS were on all four drug groups. ${ }^{8}$ The proportion of prescribed drug doses being taken (adherence) and the continuing use of these drugs over time (persistence) after discharge in this population are unknown. Observational data from a US study showed that for the period 1995-2002, use of evidence-based therapies for CHD remained suboptimal, which the authors argued was due to lack of adherence, ${ }^{2}$ an issue requiring attention. In the AusHeart primary care study, ${ }^{9}$ the sample of Australian general practitioners (GPs) who participated in 2008 reported that about $70 \%$ of patients with established cardiac disease were prescribed a statin, $70 \%$ had antiplatelet agents and about $75 \%$ used antihypertensive medication. However, adherence was not measured. More recent data from New Zealand showed a decreased use of these drugs at a median of 2.9 years after discharge following coronary artery bypass surgery, with $83 \%$ of patients remaining on aspirin, $62 \%$ on $\beta$-blockers, $72 \%$ on statins and $43 \%$ on ACEI/ARB. ${ }^{10}$

Globally, decreased morbidity and mortality have been associated with cardiovascular drug adherence using clinical trial populations ${ }^{11}$ and population-based studies. ${ }^{2} 1213$ Few studies have investigated the economic impact of nonadherence to cardiovascular preventive drugs following a cardiac event. ${ }^{14}$ Most studies have arisen from the USA and these have generally been confined to specific segments of the population, such as those with private health cover (and therefore restricted to those aged less than 65),${ }^{15}$ those eligible for 'Medicaid', or those eligible for Medicare (over 64 years of age). Other population-based studies have not included economic evaluation components, ${ }^{12}$ or have other limitations such as using adherence rates from other studies, ${ }^{16}$ investigating adherence/costs of single drugs, ${ }^{13}{ }^{17}$ or did not acquire very long-term follow-up data (more than 1-2 years) on adherence and/ or outcomes. ${ }^{15} 18$ Despite these limitations, studies evaluating the cost-effectiveness of adhering to CVD drugs have generally found adherence reduced CVD events, mortality, hospital readmissions and costs. ${ }^{19}$ To more accurately measure these benefits in a generalisable manner, our study will use a very long-term follow-up population-based study design with extensive information on previous and future medical history, and will also target the main age group requiring cardiac secondary prevention.

Some recent observational evidence contradicts conclusions based on findings from earlier randomised controlled trials. For example, a lack of significant difference in outcomes between users and non-users of $\beta$-blockers in stable outpatients with prior myocardial infarction (MI) has been reported. ${ }^{20}$ Another knowledge gap for this population is the optimum duration of dual antiplatelet therapy in patients receiving coronary artery stents. ${ }^{21}$ The population-based data sets we have established will allow us to investigate such clinical questions.

While clinical trials are generally considered the gold standard for measuring efficacy ('can it work'), restrictions imposed on clinical trial cohorts in relation to such aspects as age, comorbidities and concurrent drug use result in a lack of generalisability. Furthermore, the relatively short time frames used in clinical trials do not allow for monitoring of 'very long-term' adherence, persistence, and morbidity or mortality outcomes. Hence, the balance between benefits and risks of treatment in routine clinical practice needs to be assessed in the wider (and increasingly elderly) population in whom they are prescribed. Observational studies assess the safety and effectiveness of treatment ('does it work') and its cost-effectiveness ('is it worth it') in the actual population receiving the treatment under real-world conditions. ${ }^{22}$ Therefore, it is imperative that population-based studies are also carried out to complement results from clinical trials, particularly for very long-term follow-up and generalisability to the population.

Our study aims to investigate a number of aspects related to the use of secondary preventive medicines in people aged 65 years and over who have had their firstreported (incident) $\mathrm{CHD}, \mathrm{HF}$ or $\mathrm{AF}$ hospitalisation. This will include a description of dispensing patterns over time for commonly prescribed secondary preventive medicines with follow-up out to 8.5 years following hospitalisation. The study aims to identify clinical and sociodemographic factors influencing dispensing patterns and will also assess long-term outcomes in relation to medication adherence. Finally, the cost-effectiveness of long-term medical therapy following incident hospitalisation for $\mathrm{CHD}, \mathrm{HF}$ and $\mathrm{AF}$ will be assessed, in addition to the impact of adherence on cost-effectiveness.

\section{METHODS}

\section{Data sources}

The study is founded on government-held administrative databases that capture health-related information 
(figure 1). The Hospital Morbidity Data Collection (HMDC), Emergency Department Data Collection (EDDC) and the Mortality Register (MR) contain records of hospital admissions, emergency department visits and deaths for the population of WA, and are held by the Western Australian Department of Health. The Pharmaceutical Benefits Scheme (PBS) and Medicare Benefits Scheme (MBS) databases are held by the Australian Federal Government. The PBS database contains detailed patient-level information related to payments for medications dispensed from pharmacies (community as well as private and some public hospitals). The PBS data set also contains records from the Repatriation Pharmaceutical Benefits Scheme (RPBS), which covers pharmaceuticals dispensed to eligible veterans, war widows/widowers and their dependants. The MBS data set contains fields related to health services utilised outside of the hospital system, such as visits to GPs and specialists, and laboratory testing. Linkage of the PBS and MBS claims data with the HMDC, EDDC and MR data occurs via the WA Data Linkage System (WADLS). The WADLS uses a probabilistic matching technique to link databases based on full name, address, phonetic compression algorithms and other identifiers. The matching procedure has been estimated to be $99.9 \%$ accurate. ${ }^{23}$ It is this linkage that allows investigation of factors related to medication use and adherence, use of general practice and specialist services, outcomes and how adherence might affect future outcomes.

\section{Australian healthcare system}

The Australian healthcare system, which contains public and private services, is overseen by the State and Federal governments. Central to the Australian healthcare system is Medicare, the national health insurance provided to all permanent residents and citizens of Australia through the Federal Government. It provides free and equal access to public hospital services, in addition to rebates for out-of-hospital services including GP services and subsidies for eligible PBS medicines.
Figure 1 Variables sourced from State and Federal population-based administrative health data to create the linked study data sets (CHD, coronary heart disease; HF, heart failure; $A F$, atrial fibrillation; GP, general practitioner; SEIFA

Socio-Economic Indexes for Areas).
Hospital Morbidity Data Collection (1980-2008)

- Unique identifier

- All public and private hospitalisations in WA
Pharmaceutical Benefits Scheme (2002-2011)

- Unique identifier

- Government subsidised drugs

- Drug type and dates dispensed from pharmacies

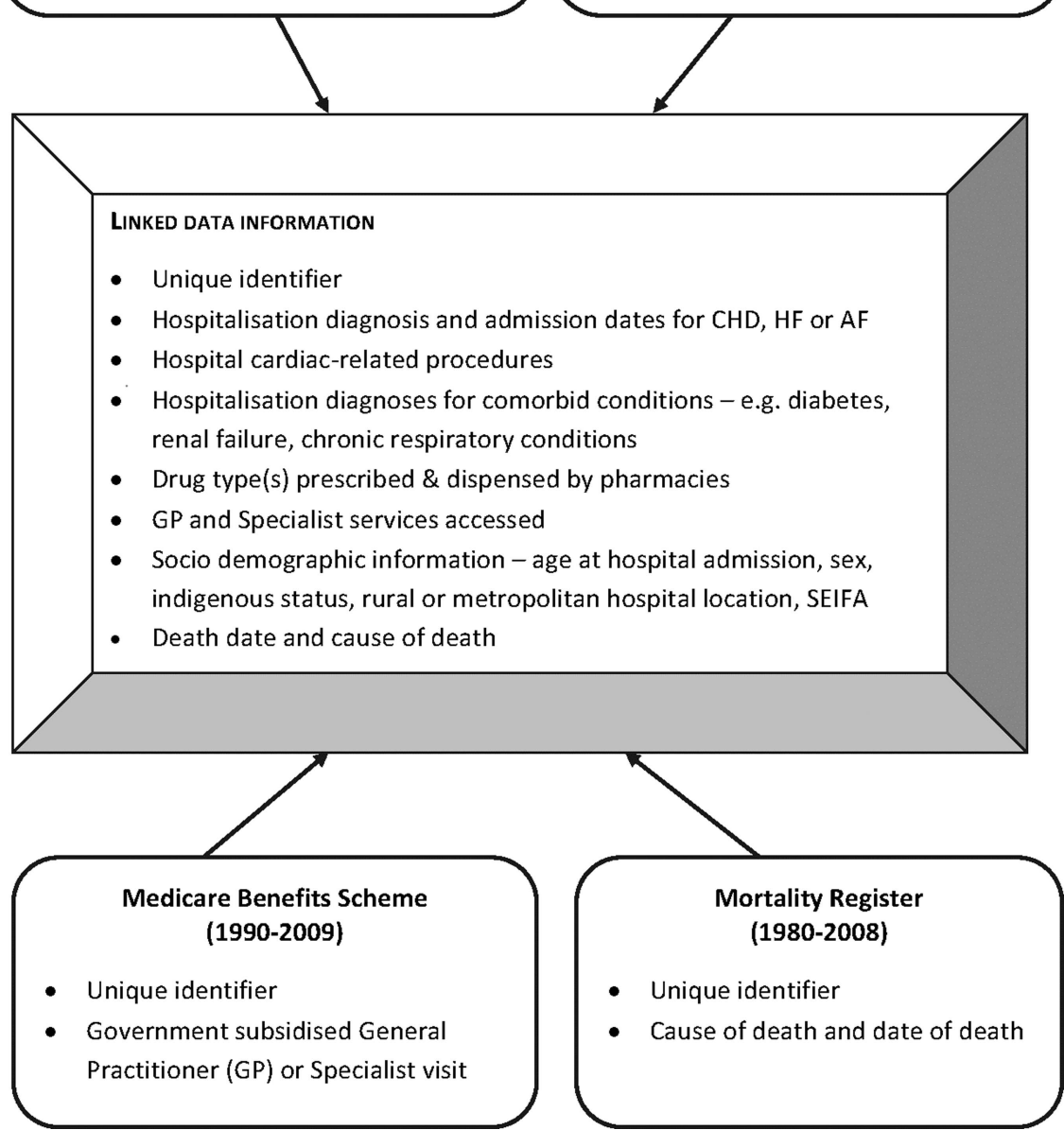


Eligible prescribed medicines that are listed on the PBS allow people to access necessary medicines for most medical conditions at a much reduced price and occasionally for free. The amount paid by the patient is known as the patient copayment, and any remaining cost is paid by the Federal Government.

Government expenditure on health accounted for $9.51 \%$ of the gross domestic product (GDP) in 2011/ $2012 .^{24}$ The costs of PBS medications are significant, at an estimated \$A9 billion for the 2012/2013 financial year, ${ }^{25}$ or $0.67 \%$ of the national GDP. ${ }^{26}$ Notably, the two most frequently dispensed drugs, which were also the most costly (in absolute terms) for the Federal Government in 2012/2013, were the lipid-lowering drugs atorvastatin and rosuvastatin. The combined annual cost to government of these two drugs during 2012/2013 was $\$$ A725.2 million. ${ }^{25}$ The dispensing of these and other common CVD preventing drugs has generally increased over the past decade (figure 2), with exceptional increases in statins and ARBs. A state-specific comparison of dispensed cardiovascular drugs (online supplementary figures S1-S5) suggests that trends over time during the study period for drugs dispensed in the state of WA are representative of trends in the other Australian states/territories.

Individuals aged 65 years or more are eligible for a concession card which allows access to PBS listed drugs at a lower patient copayment. In 2004-2005, approximately $90 \%$ of Australians aged $65-75$ years and $95 \%$ of those aged 75 years and over possessed a concession card. ${ }^{27}$ Furthermore, once a patient reaches a predetermined threshold during a given calendar year (the Medicare Safety Net), a further reduction in patient copayment applies for the remainder of that calendar year. For those on concession cards $(>90 \%$ of Australians
$65+$ years), this reduces copayments for PBS listed items to zero.

\section{Limitations of PBS and MBS data}

Many limitations of using PBS and MBS data, such as differences in government copayment allocations between those with or without concession cards, are obsolete due to our age restriction of 65 years or over. Unfortunately, the PBS database does not include the reason for being dispensed a drug, nor the dose and frequency, so the quantity taken per day will be unknown (although the drug strength is known). PBS data contain records of dispensing, not prescribing, so the prescription rates will be unknown (ie, if a drug is prescribed but not dispensed). Since aspirin is available with and without a prescription, not all aspirin use is captured in the PBS database. However, in those taking aspirin for secondary prevention, it is more cost-effective to obtain aspirin by prescription. In seniors with CHD ( $\mathrm{n}=25$ 961), pilot data showed that $75 \%$ have at least one record for aspirin ( \pm other antiplatelets) in the PBS data and $85 \%$ have at least one record for aspirin or clopidogrel (the latter is usually prescribed with aspirin). A previous analysis of drug data from medical notes in WA has shown that aspirin is well prescribed for patients discharged from hospital for MI and revascularisation procedures ${ }^{28}$ and ACS. ${ }^{8}$ Hospital discharge medications are available electronically from pharmacy dispensing databases in Perth hospitals (public and private) and will be used to check the completeness of PBS data for drugs such as aspirin. Finally, since 1999, Indigenous Australians living in remote areas have access to PBS drugs under Section 100 of the National Health Act 1953. This benefits 36\% of the Aboriginal population in Australia, ${ }^{29}$ but these are not recorded in the PBS data set.
Figure 2 Numbers of different cardiovascular drugs dispensed to Australian concession card holders during the calendar period 2002-2013. Data sourced from https://www.

medicareaustralia.gov.au/ statistics/pbs_item.shtml comprise all dispensed medications for concession card holders, including those accessed through the Medicare safety net. Dispensing numbers are based on processing dates rather than occasional artificial spikes or troughs due to administrative ACEi, ACE inhibitor; ARB, angiotensin receptor blocker; Asp or Clop, aspirin and/or clopidogrel. (accessed June 2014). Data supply dates, which may lead to issues. B-blockers, $\beta$-blockers;

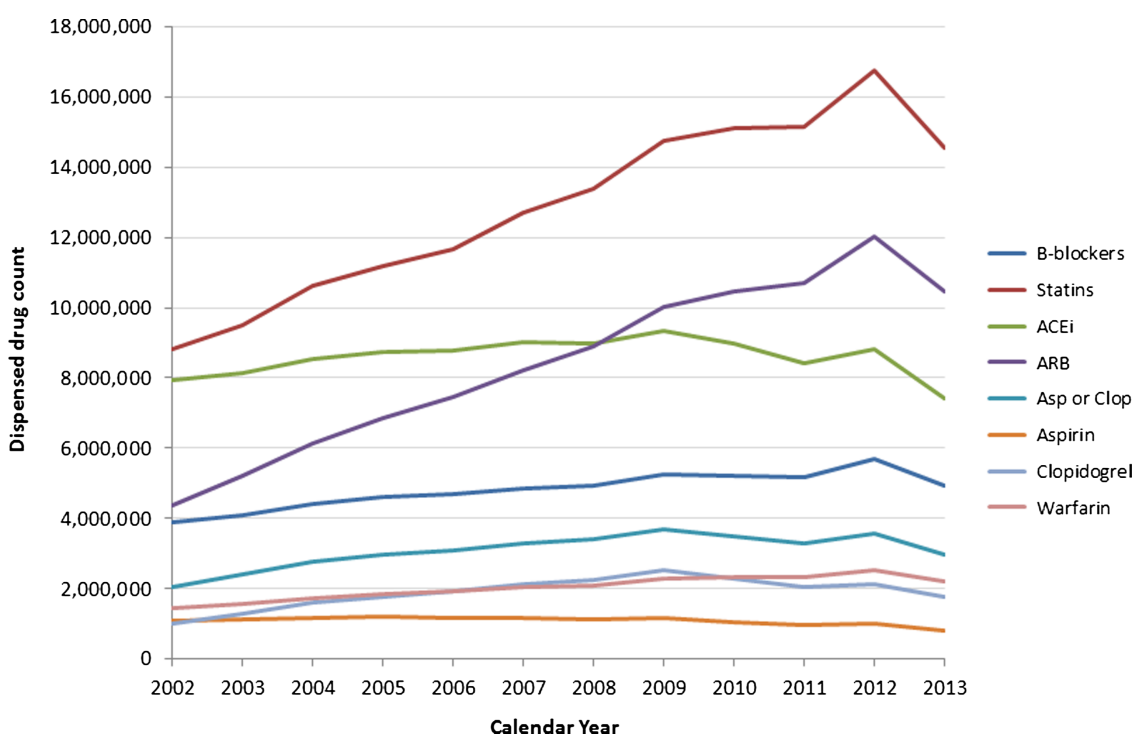


The MBS data excludes the following: (1) public patients at public hospitals (who receive free healthcare through Medicare); (2) patients with Department of Veterans' Affairs cards; (3) diagnoses and reasons for visits to medical practitioners or pathology tests; (4) item numbers may not identify individual pathology tests, but a number of like tests bundled together based on cost and (5) attendances at Aboriginal Medical Services.

\section{Study cohort}

An initial cohort of 63558 people was identified, who were admitted to any WA hospital from 2003 to the end of 2008 with a principal discharge diagnosis of CHD (International Classification of Diseases (ICD)-10-AM I20-I25), HF (I50) or AF (I48). After restriction by age, a 'seniors' study cohort was identified, which included 39748 people aged 65 years or over. The age restriction is designed to address some of the limitations of the PBS data described above, and our group has used this method previously. ${ }^{30} 31$ The majority of people in this age group are concession card holders with a lower copayment, ${ }^{27}$ which provides good coverage of PBS drugs in seniors; however, methods are needed to reduce information error arising from those with incomplete PBS data." . $^{2}$ To adjust for this, a category of 'no PBS record of any type' will be used to separate seniors likely to have missing drug data. The number of records linked to the seniors' cohort is shown in table 1 .

The initial and subsequent seniors' cohorts were identified from a source file of admissions for heart disease (I10-I52), diabetes (E10-E14) and renal failure (N17-N19) recorded in the HMDC, with all their admission records (regardless of diagnosis) extracted for the period January 1980 to December 2008. Records for the study cohort currently include HMDG data linked to deaths (to December 2008), EDDC records for 20022008 (EDDC started in 2002), PBS data (1 July 2002 to 30 June 2011) and MBS data (1 January 1990 to 31 December 2009; table 1).

We will seek updated HMDC, EDDC and death data to include records up to 2013 for existing patients in the HMDC data set. This will allow at least 5 years of follow-up (for readmissions, emergency department presentations and death) for all patients in the study cohorts. Availability of PBS data from 2002 to 2011 will provide at least 6 months before any hospital admission and at least 2.5 years after any admission.

\section{Drug dispensing patterns}

Identification of drugs dispensed to individuals will be performed using PBS item codes and Anatomical Therapeutic Chemical (ATC) codes $^{33}$ contained within the PBS data set. The drug classes to be assessed in the study will include oral anticoagulants (warfarin), antiplatelets (aspirin, clopidogrel, prasugrel, ticagrelor), $\beta$-blockers (atenolol, metoprolol, carvedilol, bisoprolol, propanolol), statins (simvastatin, rosuvastatin, pravastatin, fluvastatin, atorvastatin), ACEI (captopril, fosinopril, enalapril, ramipril, quinapril, lisinopril, trandolapril, perindopril) and ARB (irbesartan, olmesartan, candesartan, telmisartan, eprosartan).

Dispensing patterns will be fundamental to a number of components of this study. Initially, participants who are identified as having a first reported (incident) hospital admission for a cardiac event will be further classified based on: (1) prior use of the relevant cardiopreventive drugs; (2) no prior drugs of interest dispensed; (3) drugs of interest dispensed only up to 12 months prior to the cardiac admission; (4) drugs of interest dispensed after the cardiac admission and (5) drugs of interest dispensed before and after the cardiac admission. We will assess continuation or cessation of treatment (using PBS data) for a minimum period of 2.5 years (for 2008 cases) and up to 8.5 years for the 2003 cases and estimate the proportion still receiving treatment over time following the incident admission.

Prevalent cases at the midpoint (30 June) of each calendar year from 2003 to 2008 will be determined by using a fixed 20-year look-back period in the HMDC to identify surviving cases of the diseases of interest. We will then determine the proportion of prevalent cases still receiving treatment at that time as the proportion with dispensed medications within 1, 2, 3 or 6 months on either side of 30 June (to account for seasonal variations in dispensing patterns).

Clinical data will be obtained from hospital pharmacy discharge drug registers (teaching and private hospitals) and cardiology registers (from teaching hospitals). In addition, clinical data sets that the research team have established include laboratory data (cardiac biomarkers,

Table 1: Number of records linked for the 39748 patients in the Western Australian seniors' study cohort of coronary heart disease, heart failure and atrial fibrillation

\begin{tabular}{llll}
\hline Source & Core Datasets & Period covered & Total number of records \\
\hline WA - Data Linkage System & HMDC/Death & $1980-2008^{*}$ & 814997 \\
& EDDC & $2002-2008^{\star}$ & 202220 \\
Commonwealth - Dept of Health & PBS & $01 / 07 / 2002-30 / 06 / 2011$ & 16561926 \\
& MBS & $01 / 01 / 1990-31 / 12 / 2009$ & 22224407 \\
\hline
\end{tabular}

${ }^{*}$ An additional 5 years (2009-2013) of data for existing patients will be obtained.

EDDC, Emergency Department Data Collection; HMDC, Hospital Morbidity Data Collection; MBS, Medicare Benefits Schedule;

PBS, Pharmaceutical Benefits Schedule; WA, Western Australia. 
cholesterol, fasting blood glucose, glycated haemoglobin, serum creatinine, estimated glomerular filtration rate, haemoglobin) and clinical data from medical notes of teaching and private hospitals for a random sample of the cohort (includes diagnosis, inpatient and discharge drugs, ECGs, medical history, symptoms, procedures, laboratory tests).

\section{Adherence and persistence to medications}

There is a large variation in terminology describing people's propensity to take medications for the appropriate length of time at the appropriate dose. ${ }^{34}$ For this study, we will define adherence to mean the proportion of prescribed doses of medication actually taken by patients over a specified period, and persistence as the continuing use of medications from time of initiation to discontinuation of therapy. As there is no gold standard for estimating adherence using drug dispensing records, we will use several methods described by Krousel-Wood et al, ${ }^{35}$ including: (1) medication possession ratio (MPR), (2) continuous measure of medication gap (CMG) and (3) continuous single interval medication availability (CSA). These measure different aspects of adherence, and observations will be made regarding adherence using the collective interpretation of the measures. As described by Krousel-Wood et $a l,{ }^{35}$ MPR will be defined as the sum of the days' supply of medication obtained between the first and last pharmacy fill divided by the total number of days in this time period. CMG will be calculated by dividing the total number of days without medication between the first and last pharmacy fill by the number of days in this time period. ${ }^{35}$ CSA will equate to the days of supply for a medication obtained at the pharmacy fill divided by the number of days before the next pharmacy fill for the same medication. ${ }^{35}$

While a number of different adherence measures have been reported, MPR and the 'proportion of days covered' (PDC) are most widely used, and in a comparative study by Karve et al. ${ }^{36}$ MPR and PDC were suggested to be the optimal measures of choice in terms of predictive abilities, with MPR nominated in first place due to its apparently wide use in pharmacy claims-based studies. ${ }^{36}$ The method of 'refill sequence' will be used as a measure of persistence and this will accompany all measures of adherence. ${ }^{37}$

In PBS data, adherence can only be adequately measured for drugs with stable dose regimens used for extended periods (rather than episodic use). The majority of drugs of interest in this study will fit these requirements, and for those that do not (eg, warfarin), we can still estimate a measure of persistence. ${ }^{38}$ Also, patients may have stopped therapy due to side effects (eg, bleeding with clopidogrel or warfarin), so we will use the linked data to check for hospital admissions and emergency department presentations for these, as well as for changes to drug groups (eg, from ACEI to ARB). We can identify from the HMDC a history of renal failure, peptic ulcer disease, gastrointestinal bleeding and chronic respiratory conditions for possible contraindications to some of the drugs. We can also account for changes to PBS restrictions using knowledge of the dates these occurred, such as changes to prescribing of statins.

\section{Statistical analyses}

\section{Dispensing trends in discharged patients}

The proportion of people still receiving treatment over time following their incident cardiac-related hospital admission in the period 2003-2008 will be estimated using Kaplan-Meier survival methods. MPR, CMG and CSA will be calculated to assess adherence. ${ }^{35}$ Trends will be examined for both incident cases in 2003-2008 and prevalent cases identified at 30 June of each calendar year from 2003 to 2008 .

\section{Determinants of initial prescription and long-term use of cardiac medications}

The relationship between sociodemographic and clinical factors, and initial prescribing and adherence to treatment measures will be investigated using multivariable logistic and Cox regression methods. Factors to be investigated include: (1) social and demographic variables (age, sex, socioeconomic status, rural and remote residence and Indigenous status (Aboriginality) - data on Indigenous status may be missing from remote locations in PBS/MBS data and we will explore the extent of this through the HMDC); (2) clinical and health service factors including comorbid conditions (eg, diabetes, hypertension, renal failure, chronic obstructive pulmonary disease, cancer), Charlson comorbidity score (Dartmouth-Manitoba), emergency admission, coronary revascularisation, type of hospital, health insurance status, 10-year history of GP/specialist care, follow-up visits to GPs/specialists and (3) medication factors (number of evidence-based drugs, interactions with other common drugs (eg, non-steroidal antiinflammatory drugs), adverse effects of drugs requiring presentation to hospital (from the HMDC and EDDC), changes in drug group (eg, ACEI to ARB)). A Charlson comorbidity score $^{39}$ will be calculated for each incident case by applying a fixed 5-year look-back period using the HMDC. We will use the Dartmouth-Manitoba ICD code assignments ${ }^{40}$ with extended $\operatorname{codes}^{41}$ in calculating the Charlson score based on the original 17 Charlson comorbidities.

In Cox regression analyses of adherence to treatment such as time to cessation of treatment, patients will be censored at death or end of follow-up. A second analysis will censor patients at hospital presentation for the CVD event of interest (CHD, HF or AF-related stroke) to identify factors related to adherence to treatment in the period following their incident admission to their next presentation. Similarly, logistic regression methods will be used to investigate sociodemographic, disease history and clinical factors related to receipt of treatment among prevalent cases. This will provide information on the variation in prevalence of treatment according to 
sociodemographic factors and for different cardiac conditions after adjusting for the aforementioned factors.

A multivariate logistic regression model will be developed to estimate propensity to be prescribed initial medications based on the main sociodemographic and clinical determinants. The linear predictor from this model can be used as a measure of the propensity of an individual to receive initial treatment. Propensity scores will be used for control of confounding by indication when assessing the relationship between adherence to secondary prevention medications and subsequent outcomes (see next section). This will be performed in two ways, first using propensity scores as adjustment variables and second using propensity probabilities as inverse weights in Cox proportional hazards regression models with time-dependent medications exposure/adherence.

\section{Secondary preventive medicine use and clinical outcomes}

Cox regression models applied to the incident cohort will be used to determine the relationship between use of medications and major clinical outcomes including death, admission for ACS, HF and AF-related stroke for up to 11 years from the incident admission. Exposure to medications and adherence are time-varying covariates and in the Cox model the risk of a major clinical outcome at any time during follow-up can be modelled as a function of the medication use at that follow-up time or some cumulative measure of medication use over the whole period from index admission until that follow-up time. This approach is not subject to immortal time bias that can occur in pharmacoepidemiology studies. $^{42}$

Measures of aggregate health utilisation over time from index admission will be developed based on the hospital readmissions, MBS and PBS items. A linear regression or other generalised linear model (depending on the distribution of the health utilisation measure) will assess the relationship between initial prescribing and adherence to treatment over the first 2 years of follow-up on aggregate health service utilisation in survivors. These analyses will also control for the propensity to receive an initial prescription.

\section{Cost-effectiveness of long-term pharmacological treatment}

Patients will be followed for 1, 2, 5 and 11 years from hospital discharge. The primary outcome will be death (all cause and cardiovascular). Readmissions, including those for MI, ACS, HF, AF-related stroke and coronary revascularisation, may be assessed separately as costs and outcomes. Incremental cost-effectiveness ratios will address cost per life year gained for different levels of adherence. Adherence will be measured as described previously, and patients will be grouped into either optimal or suboptimal adherence. The cut-off value for these will be varied, allowing us to calculate costeffectiveness ratios relative to different levels of adherence in the population. Cost-effectiveness ratios at each level of adherence will be plotted on the cost- effectiveness plane. CIs (using bootstrapping analysis) will demonstrate variability in the ratio. We can then determine the level of adherence that needs to be achieved in the population to optimise the costeffectiveness ratio for each evidence-based drug or pharmacological group. Sensitivity analysis will assess the robustness of the cost-effectiveness ratios subject to changes in the main variables.

MBS data will be the source for determining the use of health services after discharge, including visits to GPs and specialists, and for laboratory tests. It will provide information on: (1) frequency (intensity) and regularity (periodicity) of visits to GPs and specialists after hospital discharge and (2) types of follow-up medical services. Methods developed internally ${ }^{43}$ will be used to calculate intensity and periodicity scores. Various points of entry to the person-time pool will be examined, such as: (1) fixed periods prior to the first cardiac-related admission, (2) at date of discharge and (3) on 1 January 1990 (start of the MBS data). Exit points will be: (1) fixed periods from entry (eg, 1, 2 or 5 years) and (2) 31 December 2009 (last date in the MBS data). Poisson (or negative binomial if overdispersion is present) log-linear regression models will be used to estimate rate ratios of GP and specialist visits adjusting for demographic variables, Charlson comorbidity score, year of incident admission, measure of adherence and type of drugs received.

Costs of resource use will be obtained at the individual level for a patient's hospital records, PBS and MBS data. Costs for pharmaceutical and Medicare services will be obtained from the PBS and MBS schedules corresponding to the time of service. DRG (diagnosis-related group) level costs for all WA hospital admissions will provide average costs. Costs will be adjusted to a reference year by inflating the costs from previous years using the corresponding Health Price Index obtained from the Australian Institute of Health and Welfare (AIHW). Costs and outcomes compared across differing time periods will be adjusted for time preference using the standard $3-5 \%$ discount rate. In a subgroup analysis, we will focus on teaching hospitals because we are able to obtain individual patient costs from their business units, with admission costs broken down into direct and overhead itemised costs (eg, medical, nursing, theatre, allied health).

\section{Consumer and community participation}

Community forums will be held to make research findings more relevant to consumers and to obtain feedback on issues related to adherence and persistence. The forums will use a developed process called 'community conversations'. The process involves bringing together community members to discuss a series of set questions. A 'world café' process is used, whereby a small number of participants (eg, 5-6) will be seated at each table and discuss a specific question(s) at that table, after which time there will be rotations to discuss other questions. Forums will be coordinated by the research team and experienced consumer 
representatives from the Consumer Participation Unit (School of Population Health, The University of Western Australia). Responses from participants will be transcribed into a word document and analysed using conventional content analysis $^{44}$ to provide a report for the research team. Outputs from the study will be disseminated to consumers and the community via written newsletters/articles and through various consumer/community networks and a presentation of final results will be held for consumer participants.

\section{Dissemination}

The large amount of data available, and to be obtained, will result in numerous publications of our findings in peer-reviewed medical journals. Findings will also be presented at local, national and international conferences. Relevant findings and summaries will be translated into lay language and disseminated to consumer groups following their participation in consumer forums. Additional dissemination of study outputs will be through our collaboration with NPS MedicineWise (an independent, evidence-based organisation that provides practical tools and information about medicines, health conditions and medical tests) and through the local Medicare centres and cardiologists.

\section{DISCUSSION}

The importance of population-based information on secondary preventive cardiac medicine use is vital in determining the effectiveness of commonly prescribed drugs in preventing future cardiac events and/or death. Once released into the broader population, drugs are no longer in a controlled 'clinical trial' environment and are exposed to a population that is more age and gender diverse, with numerous comorbidities and concurrent 'other drug' use, and potentially has a significant proportion of non-adhering patients. The effectiveness of secondary preventive medications in reducing CVD events is likely to be jeopardised by such issues, making it imperative that longitudinal data on adherence and associated clinical outcomes are assessed in a typical population that uses these medications. Our study will investigate drug dispensing patterns in the WA population for those having a hospitalisation for CHD, $\mathrm{HF}$ or AF. We will measure the adherence, persistence and subsequent health and cost-effectiveness of secondary preventive medications for these cardiac conditions, in addition to identifying determinants for adherence or lack thereof.

A large body of evidence supports the use of pharmaceutical agents for secondary prevention in those who have had a cardiac event. ${ }^{45}$ However, evidence suggests that adherence to these medications wanes over time, contributing to less pronounced health benefits and increased economic cost to the community. ${ }^{19}$ Indeed, several large longitudinal registry studies have highlighted the adverse effects of non-adherence on patient outcomes such as all-cause and CVD-specific mortality. ${ }^{12} 144647$

In Australia, several studies have shown reduced adherence and persistence over time to $\beta$-blockers, statins, ACEI/ARBs, antiplatelet therapy and calcium-channel blockers. ${ }^{48-50}$ In a random sample of 48690 Australian long-term health concession card holders on an ACEI, ARB and/or calcium-channel blocker, ${ }^{48} 81 \%$ of participants who had an initial prescription filled for ACEI or ARB medications had a second prescription filled, and the proportions decreased to approximately $45 \%$ and $47 \%$, respectively, for ACEIs and ARBs, after median periods of approximately 2 years. However, in a study of 9635 veterans following a hospitalisation for ischaemic heart disease, ${ }^{49}$ adherence levels of $75 \%$ to ACEI/ARBs (median treatment duration of 5.4 years), $83.9 \%$ for lipid-lowering therapies (median duration of 6.2 years) and $84 \%$ for non-aspirin antiplatelets (median duration of 5 years) were observed.

These studies suggest that non-adherence to secondary preventive cardioprotective drugs exists and varies according to drug type. The magnitude and effects nonadherence requires further investigation in terms of health outcomes and costs to the community.

Being a longitudinal population-based study, our project will be able to investigate adherence and persistence for all seniors hospitalised for CHD, HF and $\mathrm{AF}$ within WA, rather than a random sample of patients. The longitudinal data related to outcomes will allow the estimation of cause-specific and all-cause mortality as a function of adherence (and persistence). This has not been previously undertaken in Australia, and evidence linking adherence with long-term clinical outcomes is also scarce internationally. Preliminary investigation of dispensed drug trends in WA compared with the other Australian states (online supplementary figures S1-S5) suggests that the results of our study will be generalisable to the Australian population. This is clearly important due to the overlapping nature of the healthcare system (in terms of State and Federal funding) and the intervention and treatment strategies. It will also provide an evidence base for other individual Australian states.

In addition to the population-based nature of our study and the ability for a very long-term follow-up of clinical outcomes (up to 8.5 years), there are a number of other strengths of this study. A key strength is the ability to identify cardiovascular and other hospitalisations within 20 years prior to the cardiac event of interest. This will allow prior and current health to be taken into account during the analysis, which is particularly important due to the 'healthy adherer effect' concerns that are sometimes raised ${ }^{1419}$ as a limitation of studies investigating outcomes associated with drug adherence. Although the use of records for dispensing of drugs does not necessarily equate to drug ingestion, it is considered that prescription refill rates accurately reflect overall adherence in a closed pharmacy system. ${ }^{1436}$ The 
data used in our study are likely to fit this criterion (of a closed pharmacy system) given the Australia-wide coverage of records from PBS data, along with the higher subsidies for medications in those aged 65 years and over.

\section{CONCLUSION}

There is a need for long-term studies capable of measuring the adherence and subsequent health and costeffectiveness of secondary preventive medications for major cardiac disease in the population. Our ability to capture cardiac events, comorbidities and mortality within the WA population in addition to their medication use will allow us to address questions around the effectiveness of these drugs in the broader population, taking into account a measure of adherence. If threshold levels of adherence are identified, below which therapy becomes less effective, a target can be set for intervention strategies aimed at maintaining at least a minimal level of adherence and the associated beneficial health outcomes. This information will be of benefit to policymakers, consumers and healthcare providers to achieve sustainable secondary prevention for heart disease in the population.

\author{
Author affiliations \\ ${ }^{1}$ School of Population Health, The University of Western Australia, Crawley, \\ Western Australia, Australia \\ ${ }^{2}$ Western Australian Centre for Rural Health, The University of Western \\ Australia, Crawley, Western Australia, Australia \\ ${ }^{3}$ School of Medicine \& Pharmacology, Sir Charles Gairdner Hospital Unit, The \\ University of Western Australia, Nedlands, Western Australia, Australia \\ ${ }^{4}$ Department of Cardiology, Royal Perth Hospital, Perth, Western Australia, \\ Australia \\ ${ }^{5}$ St Vincent's Clinical School, University of New South Wales, Darlinghurst, \\ New South Wales, Australia \\ ${ }^{6}$ Medicinelnsight, NPS MedicineWise, Sydney, New South Wales, Australia \\ ${ }^{7}$ Consumer and Community Advisory Council, School of Population Health, \\ The University of Western Australia, Crawley, Western Australia, Australia \\ ${ }^{8}$ Health Consumers' Council (WA) Inc., Perth, Western Australia, Australia \\ ${ }^{9}$ Clinical Modelling Unit, Western Australian Department of Health, Perth, \\ Western Australia, Australia
}

Acknowledgements The authors thank the WA Department of Health and the Australian Department of Health for providing the cross-jurisdictional linked data used in this study. They are furthermore grateful to the Data Linkage Branch and Data Custodians of the WA Department of Health for providing the linked HMDC, EDDC and death data.

Contributors ASG wrote the first draft of the manuscript and will perform data cleaning/validation. The study was conceived by FMS, MSTH and MWK, with all authors contributing to the study design and planning. Analytical/ methodology design will be addressed by multiple authors. Biostatistical and epidemiological methods will be refined by MWK, FMS, MSTH, ASG, MG, MO, LN, QM, TGB, JMK and DL. Burden of disease and rural/Aboriginal health will be assessed by JMK and DL. Health economics components will be informed and supervised by EG, with analytical contribution from CG and EA. Clinical input towards design, analysis and interpretation of results will be provided by cardiologists JH and JMR. Consumer forums will be assisted by $\mathrm{AC}$ and MM. All authors provided critical feedback during manuscript development and approved the final manuscript.

Funding This research is supported by a project grant from the National Health and Medical Research Council of Australia (NHMRC project grant 1066242)
Competing interests None.

Ethics approval Human Research Ethics Committee approvals have been obtained from the Western Australian Department of Health (\#2011/62); Australian Department of Health (XJ-16), The University of Western Australia (RA/4/1/1130); participating hospitals where individual electronic hospital registries/medical notes will be accessed ( 3 public teaching, 1 public/private and 4 private and WA Country Health Service); and the Western Australian Aboriginal Health Ethics Committee (381-01/2011).

Provenance and peer review Not commissioned; internally peer reviewed.

Data sharing statement We will consider requests for data sharing on an individual basis, with an aim to sharing data whenever possible for appropriate research purposes. However, the research project uses secondary (third party) data derived from Australian (State or Federal) government registries, which are ultimately governed by their ethics committees and data custodians. Therefore, any requests to share these data will be subject to formal approval from their ethics committees overseeing the use of these data sources, along with the data custodian(s) for the data of interest.

Open Access This is an Open Access article distributed in accordance with the Creative Commons Attribution Non Commercial (CC BY-NC 4.0) license, which permits others to distribute, remix, adapt, build upon this work noncommercially, and license their derivative works on different terms, provided the original work is properly cited and the use is non-commercial. See: http:// creativecommons.org/licenses/by-nc/4.0/

\section{REFERENCES}

1. Alexander KP, Peterson ED, Granger CB, et al. Potential impact of evidence-based medicine in acute coronary syndromes: insights from GUSTO-Ilb. J Am Coll Cardiol 1998;32:2023-30.

2. Newby LK, Allen LaPointe NM, Chen AY, et al. Long-term adherence to evidence-based secondary prevention therapies in coronary artery disease. Circulation 2006;113:203-12.

3. Krum H, Jelinek MV, Stewart S, et al. 2011 update to National Heart Foundation of Australia and Cardiac Society of Australia and New Zealand Guidelines for the prevention, detection and management of chronic heart failure in Australia, 2006. Med J Aust 2011;194:405-9.

4. Camm AJ, Kirchhof P, Lip GY, et al.; European Heart Rhythm A, European Association for Cardio-Thoracic S. Guidelines for the management of atrial fibrillation: the Task Force for the Management of Atrial Fibrillation of the European Society of Cardiology (ESC). Europace 2010;12:1360-420.

5. National Heart Foundation of Australia and the Cardiac Society of Australia and New Zealand. Reducing risk in heart disease 2007 (updated 2008). Canberra: National Heart Foundation of Australia, 2008. http://www.heartfoundation.org.au/SiteCollectionDocuments/ Reduce-risk-in-heart-disease-guideline.pdf

6. Amerena JV, Walters TE, Mirzaee S, et al. Update on the management of atrial fibrillation. Med J Aust 2013;199:592-7.

7. Redfern J, Hyun K, Chew DP, et al. Prescription of secondary prevention medications, lifestyle advice, and referral to rehabilitation among acute coronary syndrome inpatients: results from a large prospective audit in Australia and New Zealand. Heart 2014;100:1281-8.

8. Gausia K, Katzenellenbogen JM, Sanfilippo FM, et al. Evidence-based prescribing of drugs for secondary prevention of acute coronary syndrome in Aboriginal and non-Aboriginal patients admitted to Western Australian hospitals. Intern Med J 2014;44:353-61.

9. Heeley EL, Peiris DP, Patel AA, et al. Cardiovascular risk perception and evidence-practice gaps in Australian general practice (the AusHEART study). Med J Aust 2010;192:254-9.

10. Looi KL, Chow KL, Looi JL, et al. Under-use of secondary prevention medication in acute coronary syndrome patients treated with in-hospital coronary artery bypass graft surgery. N Z Med J 2011;124:6-10.

11. Simpson SH, Eurich DT, Majumdar SR, et al. A meta-analysis of the association between adherence to drug therapy and mortality. BMJ 2006;333:15.

12. Rasmussen JN, Chong A, Alter DA. Relationship between adherence to evidence-based pharmacotherapy and long-term mortality after acute myocardial infarction. JAMA 2007;297:177-86.

13. Wei L, Wang J, Thompson $\mathrm{P}$, et al. Adherence to statin treatment and readmission of patients after myocardial infarction: a six year follow up study. Heart 2002;88:229-33.

14. Ho PM, Bryson CL, Rumsfeld JS. Medication adherence: its importance in cardiovascular outcomes. Circulation 2009;119:3028-35. 
15. Pittman DG, Tao Z, Chen W, et al. Antihypertensive medication adherence and subsequent healthcare utilization and costs. $A m \mathrm{~J}$ Manag Care 2010;16:568-76.

16. Hovstadius B, Petersson G. Non-adherence to drug therapy and drug acquisition costs in a national population-a patient-based register study. BMC Health Serv Res 2011;11:326.

17. Shalev V, Chodick G, Silber H, et al. Continuation of statin treatment and all-cause mortality: a population-based cohort study. Arch Intern Med 2009;169:260-8.

18. Sun SX, Ye X, Lee KY, et al. Retrospective claims database analysis to determine relationship between renin-angiotensin system agents, rehospitalization, and health care costs in patients with heart failure or myocardial infarction. Clin Ther 2008;30(Pt 2):2217-27.

19. Bitton A, Choudhry NK, Matlin OS, et al. The impact of medication adherence on coronary artery disease costs and outcomes: a systematic review. Am J Med 2013;126:357.e7-27.

20. Bangalore S, Steg G, Deedwania P, et al. B-blocker use and clinical outcomes in stable outpatients with and without coronary artery disease. JAMA 2012;308:1340-9.

21. Cassese S, Byrne RA, Tada T, et al. Clinical impact of extended dual antiplatelet therapy after percutaneous coronary interventions in the drug-eluting stent era: a meta-analysis of randomized trials. Eur Heart J 2012;33:3078-87.

22. Järvinen TLN, Sievänen $H$, Kannus $P$, et al. The true cost of pharmacological disease prevention. BMJ 2011;342:d2175.

23. Holman CD, Bass AJ, Rouse IL, et al. Population-based linkage of health records in Western Australia: development of a health services research linked database. Aust N Z J Public Health 1999;23:453-9.

24. AlHW. Australian Institute of Health and Welfare. Health expenditure Australia 2011-12. Health and welfare expenditure series no 50 Cat no HWE59. Canberra: AlHW, 2013.

25. PBS Information Management Section (Department of Health) Expenditure and prescriptions twelve months to 30 June 2013. 2013.

26. Sweeny K. The impact of further PBS reforms: report to medicines Australia. Centre for Strategic Economic Studies. Victoria University. Melbourne, Australia, 2013.

27. ABS. Household expenditure on health: a snapshot, 2004-05. Canberra: Australian Bureau of Statistics (ABS) cat no. 4836.0.55.001, 2008

28. Briffa T, Hickling S, Knuiman M, et al. Long term survival after evidence based treatment of acute myocardial infarction and revascularisation: follow-up of population based Perth MONICA cohort, 1984-2005. BMJ 2009;338:b36.

29. Kelaher M, Taylor-Thomson D, Harrison N, et al. Evaluation of PBS medicine supply arrangements for remote area Aboriginal Health Services under S100 of the National Health Act. Canberra: Department of Health and Ageing, 2004

30. Gunnell AS, Einarsdottir K, Sanfilippo F, et al. Improved long-term survival in patients on combination therapies following an incident acute myocardial infarction: a longitudinal population-based study. Heart 2013;99:1353-8.

31. Price SD, Holman CD, Sanfilippo FM, et al. Use of case-time-contro design in pharmacovigilance applications: exploration with high-risk medications and unplanned hospital admissions in the Western Australian elderly. Pharmacoepidemiol Drug Saf 2013;22: $1159-70$
32. Mai Q, Holman CDA, Sanfilippo F, et al. Mental illness related disparities in diabetes prevalence, quality of care and outcomes: a population-based longitudinal study. BMC Med 2011;9:118.

33. WHO. WHO Collaborating Centre for Drug Statistics Methodology, Guidelines for ATC classification and DDD assignment 2014. Oslo, Norway: World Health Organisation, 2013.

34. Cramer JA, Roy A, Burrell A, et al. Medication compliance and persistence: terminology and definitions. Value Health 2008;11:44-7.

35. Krousel-Wood M, Islam T, Webber LS, et al. New medication adherence scale versus pharmacy fill rates in seniors with hypertension. Am J Manag Care 2009;15:59-66.

36. Karve S, Cleves MA, Helm M, et al. An empirical basis for standardizing adherence measures derived from administrative claims data among diabetic patients. Med Care 2008;46: 1125-33.

37. Caetano PA, Lam JM, Morgan SG. Toward a standard definition and measurement of persistence with drug therapy: examples from research on statin and antihypertensive utilization. Clin Ther 2006;28:1411-24; discussion 10

38. Ortiz MS, Calcino G, Germanos P. Compliance with warfarin therapy by Australian patients with atrial fibrillation. Value Health 2012;15: A376.

39. Charlson ME, Pompei P, Ales KL, et al. A new method of classifying prognostic comorbidity in longitudinal studies: development and validation. J Chronic Dis 1987;40:373-83.

40. Romano PS, Roos LL, Jollis JG. Adapting a clinical comorbidity index for use with ICD-9-CM administrative data: differing perspectives. J Clin Epidemiol 1993;46:1075-9; discussion 81-90.

41. Quan $\mathrm{H}$, Sundararajan $\mathrm{V}$, Halfon $\mathrm{P}$, et al. Coding algorithms for defining comorbidities in ICD-9-CM and ICD-10 administrative data. Med Care 2005;43:1130-9.

42. Suissa S. Immortal time bias in pharmacoepidemiology. Am J Epidemiol 2008;167:492-9.

43. Mai Q. The use, quality and outcomes of primary health care services in Western Australian mental health clients: a population-based longitudinal study [PhD]. The University of Western Australia, 2011.

44. Hsieh H-F, Shannon SE. Three approaches to qualitative content analysis. Qual Health Res 2005;15:1277-88.

45. Fleg JL, Aronow WS, Frishman WH. Cardiovascular drug therapy in the elderly: benefits and challenges. Nat Rev Cardiol 2011;8:13-28.

46. Ho PM, Magid DJ, Shetterly SM, et al. Medication nonadherence is associated with a broad range of adverse outcomes in patients with coronary artery disease. Am Heart J 2008;155:772-9.

47. Kumbhani DJ, Steg PG, Cannon CP, et al. Adherence to secondary prevention medications and four-year outcomes in outpatients with atherosclerosis. Am J Med 2013;126:693-700.e1.

48. Simons LA, Ortiz M, Calcino G. Persistence with antihypertensive medication: Australia-wide experience, 2004-2006. Med J Aust 2008;188:224-7.

49. Roughead EE, Vitry Al, Preiss AK, et al. Assessing overall duration of cardiovascular medicines in veterans with established cardiovascular disease. Eur J Cardiovasc Prev Rehabil 2010;17:71-6.

50. Simons LA, Ortiz M, Calcino $\mathrm{G}$. Long-term persistence with statin therapy: experience in Australia 2006-2010. Aust Fam Physician 2011;40:319-22. 